\title{
DUAL CROSSING SYMMETRIC MODELS WITH REGGE CUTS
}

\author{
R.W. TUCKER* \\ University of Massachusetts, Amherst, Massachusetts 01002 \\ and \\ W.J. ZAKRZEWSKI \\ University of Michigan, Ann Arbor, Michigan 48108
}

Received 20 September 1971

\begin{abstract}
We investigate a class of dual crossing symmetric models which generate an infinite series of Regge cuts. The amplitudes contain Regge poles dual to finite width resonances and multi-reggeon cuts dual to multi-resonance cuts. We discuss the analy ticity and asymptotic behaviour associated with these models and indicate how the pomeron might be incorporated.
\end{abstract}

\section{INTRODUCTION}

The concept of duality [1], introduced only a few years ago, has helped us to increase our understanding of strong interaction physics and plays an important role in most of the theoretical considerations and construction of new models [2]. The Veneziano model, probably the most successful and certainly the best known and studied of these, is a simple, though ingenious and elegant representation of one particular choice of duality: duality between resonances and Regge poles [3]. The model has attracted considerable interest and has led to much research, the construction of a dual field theory [4], new ways of looking at some bootstrap problems and has even altered our phenomenological understanding of hadron physics [5]. It should be added, however, that the phenomenology usually supplements the Veneziano model with extra assumptions which break some important properties of the original model. Most of the phenomenological problems with the Veneziano model stem from an incorrect analytic structure in which all the resonances appear as bound states (poles on the real axis) and not as poles on secondary sheets in the complex plane. Although the perturbative unitarisation procedure based on the dual field theory transforms these bound states to resonance poles, this does not help phenom-

\footnotetext{
* Present adress: High Energy Physics, Cavendish Laboratory, Cambridge, England.
} 
enology as the expressions obtained are far too complicated for any comparison with experiment. Phenomenology needs relatively simple expressions which have as many desirable properties as possible and yield to calculations connected with reality.

Phenomenologically, Regge cuts are important [5]. There is still much discussion and controversy as to their form and detailed properties but their necessity seems well established now and their existence little disputed. Various types of Regge cut exist in the literature. Of these the most popular are absorptive cuts (strong [6] and weak [7]), A.F.S. [8], eikonal [9] or fixed cuts. Some methods introduce a whole series of cuts, others introduce effective cuts to simulate the whole series.

The perturbative unitarisation of the Veneziano model based on the dual field theory also leads to Regge cuts [10].

Recently interest has arisen in the construction of models which have more of the correct analytic structure associated with scattering amplitudes and which could be of some interest phenomenologically $[11,12]$. The interest centers primarily on the shifting of the resonances onto unphysical sheets (without the introduction of ancestors) and also making the amplitude possess double spectral functions with the correct boundaries. This analy tic structure usually follows from unitarity. Although it is difficult to impose unitarity it is believed that the introduction of as much of the correct analyticity as possible helps through the appropriate choice of free parameters to obtain amplitudes which minimize the effects associated with the lack of complete unitarity. In practice, introducing the appropriate structure is quite difficult and non-unique, primarily because the correct analytic structure is not completely understood, particularly in a theory with duality.

Phenomenologically we know that Regge cuts are necessary for the description of high energy scattering in terms of $t$-channel exchanges [5]. On the other hand, so far we seem to be able to describe low energy scattering adequately in terms of resonances only. It appears that we need not introduce any significant $s$-channel cuts associated with multiresonance production. However, we expect that such cuts exist even though we are not sure about the form of their discontinuities.

What are the Regge cuts dual to? Are they dual to these s-channel cuts or should we not dissociate the Regge poles from the Regge cuts in the $t$-channel when we consider duality? We believe that these questions are of great interest. One way to study such questions is by trying to construct models which exhibit the improved analytic structure that we have discussed and which also introduce Regge cuts so that we can study their structure and the properties that they impose on the scattering amplitude. Of course if the model is phenomenologically tractable a certain amount of information can be gained from comparison with experimental data although the non-uniqueness of such models makes the study of the correlations implied by the model perhaps more important at the moment*.

In this paper we introduce a class of dual, crossing symmetric models containing

\footnotetext{
* One of us (W.J.Z.) would like to thank Dr. H. Harari for an interesting discussion and for stressing this point.
} 
an infinite series of Regge cuts (multi-reggeon exchange) which simulate the effects of absorption by the inclusion of intermediate states of many particles. All models exhibit a direct duality structure, i.e., resonances are dual to Regge poles, two particle s-channel cuts build up two-reggeon exchange contributions etc. It is also shown how the introduction of the non-linear parts of the Regge trajectories enables us to construct amplitudes with the expected double spectral function boundaries. In sects. 2 and 3 we discuss the models in detail, analyzing their asymptotic behaviour and singularity structure. We show that at high energy each multi-reggeon contribution to the amplitude yields the expected asymptotic behaviour associated with the exchange of $n$ reggeons in the cross channel. In the energy-plane we find cuts which occur at energies above that of the elastic threshold for the scattering process under consideration. For rising trajectories with finite widths these cuts lie on unphysical sheets and may be associated with inelastic thresholds corresponding to multi-resonance production. In section four an attempt is made to construct a model which contains Regge-many pomeron cuts. A general discussion of these models is made in the last section.

\section{MANY REGGEON AMPLITUDE AND ITS PROPERTIES}

We consider the invariant amplitude describing the scattering of spinless particles that do not couple to the pomeron in any channel. Whenever a Regge trajectory can be exchanged, a Regge-pomeron cut can in general also be exchanged. Such cuts will be introduced in section four. Here we shall introduce only the cuts which arise from the combining of many reggeons together.

We propose the following integral representation for the amplitude valid in the region $s<0, t<0$ in terms of two dimensional anti-Euclidean vectors $\boldsymbol{q}_{s}$ and $\boldsymbol{q}_{\boldsymbol{t}}$ where $\boldsymbol{q}_{s}^{2}=-s, \boldsymbol{q}_{t}^{2}=-t$ :

$M(s, t)=\int_{0}^{1} \mathrm{~d} x[x(1-x)]^{-3} G(x, 1-x, s, t) \quad \mathrm{d}^{2} \boldsymbol{b}_{s} \quad \mathrm{~d}^{2} \boldsymbol{b}_{t} \mathrm{e}^{i \boldsymbol{b}_{s} \cdot q_{s}+i b_{t} \cdot a_{t} H(\delta),}$

where

$$
\begin{aligned}
& \delta\left(x, b_{s}, b_{t}\right)=\frac{-\lambda}{(2 \pi)^{4}} \int \mathrm{d}^{2} x_{s} \mathrm{~d}^{2} x_{t} \mathrm{e}^{-i x_{s} b_{s}-i x_{t} \cdot b_{t}} F\left(x_{s}^{2}(1-x), x_{t}^{2} x, x,(1-x)\right) \\
& \quad \times x^{-\alpha} \mathrm{R}^{\left(-x_{s}^{2}\right)+2}(1-x)^{-\alpha} \mathrm{R}^{\left(-x_{t}^{2}\right)+2}
\end{aligned}
$$

The functions $F, G, H$ are chosen as follows:

$$
H(\delta)=\mathrm{e}^{\delta}-1,
$$




$$
\begin{aligned}
& F\left(x_{s}^{2}(1-x), x_{t}^{2} x, x, 1-x\right)=f\left(x_{s}^{2}(1-x), x\right) f\left(x_{t}^{2} x, 1-x\right) \\
& \quad \times x^{-\alpha_{\mathrm{I}}\left(-x_{s}^{2}(1-x)\right)}(1-x)^{-\alpha_{\mathrm{I}}\left(-x_{t}^{2} x\right)}, \\
& G(x, 1-x, s, t)=1 .
\end{aligned}
$$

In this way finite width resonances have been incorporated by the method of Cohen-Tannoudji et al.[11]. By writing the leading Regge trajectory (which is expected to satisfy a dispersion relation) in the form

$$
\alpha(s)=\alpha_{\mathrm{R}}(s)+\alpha_{\mathrm{I}}(s),
$$

and applying the $t-x$ duality concept to the non-linear* part $\alpha_{\mathrm{I}}(s)$ of $\alpha(s)$. The form of $\alpha_{\mathrm{I}}(s)$ is determined by detailed dynamics. A simple form would be $\gamma\left(4 \mu^{2}-s\right)^{\bar{\nu}}$ where $\nu=\alpha_{\mathrm{R}}\left(4 \mu^{2}\right)+\frac{1}{2}$ in terms of the first elastic threshold. Before proceeding we must show that eq.(2.1) is convergent for $s, t<0$. For simplicity we shall take $f=1$ and $\alpha_{I}=0$ here. Then we can perform an angular integrations and obtain:

$$
\begin{aligned}
& M(s, t)=(2 \pi)^{2} \int_{0}^{1} \mathrm{~d} x[x(1-x)]^{-3} \int_{0}^{\infty} b_{s} \mathrm{~d} b_{s} \int_{0}^{\infty} b_{t} \mathrm{~d} b_{t} J_{0}\left(b_{s} q_{s}\right) J_{0}\left(b_{t} q_{t}\right) \\
& \quad \times\left[\operatorname { e x p } \left(\frac{-\lambda}{(2 \pi)^{2}} \int_{0}^{\infty} x_{s} \mathrm{~d} x_{s} \int_{0}^{\infty} x_{t} \mathrm{~d} x_{t} J_{0}\left(x_{s} b_{s}\right) J_{0}\left(x_{t} b_{t}\right) x^{-\alpha_{1}\left(-x_{s}^{2}\right)+2}\right.\right. \\
& \left.\left.\quad \times(1-x)^{-\alpha_{2}\left(-x_{t}^{2}\right)+2}\right\}-1\right]
\end{aligned}
$$

in terms of the zeroth order Bessel function. With $\alpha_{i}(s)=a_{i}+r_{i} s$ we can perform the $x_{s}$ and $x_{t}$ integrations:

$$
\begin{aligned}
& M(s, t)=(2 \pi)^{2} \int_{0}^{1} \mathrm{~d} x[x(1-x)]^{-3} \int_{0}^{\infty} b_{s} \mathrm{~d} b_{s} \int_{0}^{\infty} b_{t} \mathrm{~d} b_{t} J_{0}\left(b_{s} q_{s}\right) J_{0}\left(b_{t} q_{t}\right) \\
& \times\left[\operatorname { e x p } \left(\frac{-\lambda x^{2-a_{1}}(1-x)^{2-a_{2}}}{(2 \pi)^{2} 4 r_{1} r_{2} \ln x \ln (1-x)}\right.\right. \\
& \left.\left.\quad \times \exp \left(\frac{b_{s}^{2}}{4 r_{1} \ln x}+\frac{b_{t}^{2}}{4 r_{2} \ln (1-x)}\right)\right\}-1\right] .
\end{aligned}
$$

\footnotetext{
* This method of separating $\alpha(s)$ into linear and non linear parts has also been suggested by several authors (ref. [14]).
} 
The $b_{s}$ and $b_{t}$ integrations are convergent since for large $b_{s}$ and $b_{t}$

$$
\left|J_{0}\left(b_{s} q_{s}\right) J_{0}\left(b_{t} q_{t}\right)\right| \leqslant\left(\frac{1}{q_{s} q_{t}}\right)^{\frac{1}{2}}\left(\frac{1}{b_{s} b_{t}}\right)^{\frac{1}{2}}
$$

and the factor

$$
\exp \left\{A \exp \left[\frac{b_{s}^{2}}{4 r_{1} \ln x}+\frac{b_{t}^{2}}{4 r_{2} \ln (1-x)}\right]\right\}-1
$$

guarantees convergence with $0 \leqslant x \leqslant 1$. It is evident that $M(s, t)$ is crossing symmetric and, upon expansion of the exponential in eq.(2.3), expressible as an infinite series of terms, the first of which is the generalized Veneziano amplitude for two body scattering containing Regge poles in each channel.

We write

$$
M(s, t)=\sum_{n=1}^{\infty} M_{n}(s, t)
$$

$M_{1}(s, t)=-\lambda \int_{0}^{1} \mathrm{~d} x f(s(1-x)) f(t x) x^{-\alpha_{\mathrm{R}_{1}}(s)-\alpha_{\mathrm{I}_{1}}(s(1-x))-1}(1-x)^{-\alpha_{\mathrm{R}_{2}}(t)-\alpha_{\mathrm{I}_{2}}(t x)-1}$

Furthermore, with non-linear trajectories, this first term when appropriately continued to $s, t>0$ has a double discontinuity in a domain which may be chosen to coincide with the support of the lowest lying Mandelstam double spectral function. We shall show later how the other terms generate double discontinuities with higher lying boundaries.

The second term in the expansion of the exponential may be written

$$
\begin{aligned}
& M_{2}(s, t)=\frac{\lambda^{2}}{2 !} \int_{0}^{1} \frac{\mathrm{d} x}{(2 \pi)^{4}} \int \mathrm{d}^{2} x_{s} \int \mathrm{d}^{2} x_{t} f\left(x_{s}^{2}(1-x)\right) f\left(x_{t}^{2} x\right) \\
& X x^{\left.-\alpha_{\mathrm{R}_{1}}{ }^{\left(-x_{s}\right.}{ }^{2}\right)-\alpha_{\mathrm{R}_{1}}\left(-\left(q_{s}-x_{s}\right)^{2}\right)+1-\alpha_{\mathrm{I}_{1}}\left(-x_{s}{ }^{2}(1-x)\right)-\alpha_{\mathrm{I}_{1}}{ }^{\left(-\left(q_{s}-x_{s}\right)^{2}(1-x)\right)}}
\end{aligned}
$$

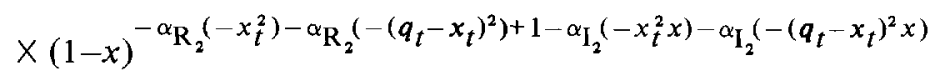

In order to simplify the analysis of the properties of this and higher terms we shall make the approximation of using linear trajectories $\alpha(s)=\alpha_{1}(s)=\alpha_{2}(s)=a+r s$ and also set $f=1$. We can then perform the two dimensional integrations in $M_{2}$ and obtain

$$
M_{2}(s, t)=\frac{\lambda^{2}}{128 \pi^{2} r} \int_{0}^{1} \mathrm{~d} x \frac{x^{1-2 a-\frac{1}{2} r s}(1-x)^{1-2 a-\frac{1}{2} r t}}{\ln x \ln (1-x)}
$$


In order to elucidate the behaviour of this integral for large $-t$ we change variable to $u=-t x$ and find

$$
M_{2}(s, t) \underset{t \rightarrow-\infty}{\sim}-\frac{(-t)^{2 a+\frac{1}{2} r s-1}}{\ln (-t) 128 \pi^{2} r} \int_{0}^{\infty} u^{-2 a-\frac{1}{2} s r} e^{-\frac{1}{2} u r} \mathrm{~d} u .
$$

Writing $\alpha_{2}(s)=2 a+\frac{1}{2} r s-1=2 \alpha\left(\frac{1}{4} s\right)-1$ we see that for $t \rightarrow-\infty$ we have

$$
M_{2}(s, t) \underset{t \rightarrow-\infty}{\sim} \frac{\lambda^{2}}{128 \pi^{2} r} \Gamma\left(-\alpha_{2}(s)\right) \frac{\left(-\frac{1}{2} t r\right)^{\alpha_{2}(s)}}{\ln (-t)} .
$$

If we analyse the $n$th term in the expansion in a similar way we see that its asymptotic behaviour is given by

$$
M_{n}(s, t) \underset{t \rightarrow-\infty}{\sim} \frac{(-\lambda)^{n}}{n !} \frac{\Gamma\left(-\alpha_{n}(s)\right)}{\ln ^{n-1}(-t)}\left(-\frac{t r}{n}\right)^{\alpha_{n}(s)},
$$

where

$$
\alpha_{n}(s)=n \alpha\left(\frac{s}{n^{2}}\right)-n+1
$$

To obtain Regge behaviour for $|t| \rightarrow \infty$ we have to perform an analy tic continuation. In the case of linear trajectories the analytic continuation of an expression such as eq.(2.9) can be readily done by "the rotation of the integration contour method" [15]. Indeed, if we change the variable with $(1-x)=\mathrm{e}^{-y}$ and then rotate the $y$ contour this method shows that the asymptotic behaviour in eqs.(2.10) and (2.11) holds for $|t| \rightarrow \infty$ everywhere except on the positive real axis. The existence of the nonlinear part to the trajectory helps to remove this restriction.

We next analyse the singularities that arise from $M_{n}(s, t)(n>1)$ in the finite positive $s$ or $t$ plane. In the $s$-channel, for example, they arise from the end point $x=0$ of the integral

$$
\begin{aligned}
M_{n} & \propto \frac{(-\lambda)^{n}}{n !} \int_{0}^{1} \mathrm{~d} x \frac{x^{-n a-\frac{r s}{n}+2 n-3}(1-x)^{-n a-\frac{r t}{n}+2 n-3}}{[\ln x \ln (1-x)]^{n-1}} \\
& =\frac{(-\lambda)^{n}}{n !} \int_{0}^{1} \mathrm{~d} x \frac{x^{-\alpha} n^{(s)+n-2}(1-x)^{-\alpha} n^{(t)+n-2}}{[\ln x \ln (1-x)]^{n-1}}
\end{aligned}
$$

We exhibit the singularities by observing that they are contained in the integral

$$
\int_{0}^{\epsilon} \mathrm{d} x \frac{x^{-\alpha} n^{(s)-1+k}}{(-\ln x)^{n-1}}, n \neq 1, k=0,1,2, \ldots
$$


where we have expanded the integrand about the lower limit of integration. To examine this integral we change variables to $u=-\ln x$ and express it in terms of the incomplete gamma function [16]

$$
\int_{-\ln \epsilon}^{\infty} \mathrm{d} u \mathrm{e}^{u\left(\alpha_{n}(s)-k\right)} u^{-n-1}=\left(k-\alpha_{n}(s)\right)^{n-2} \Gamma\left(2-n, \ln \epsilon\left(\alpha_{n}(s)-k\right)\right)
$$

The incomplete gamma function is now written in the form

$$
\Gamma(-n, x)=\frac{(-1)^{n}}{n !}\left[\Gamma(0, x)-\mathrm{e}^{-x} \sum_{m=0}^{n-1}(-1)^{m} \frac{m !}{x^{m+1}}\right]
$$

where $\Gamma(0, x) \sim \ln x$ at $x \sim 0$.

We see that eq.(2.14) behaves as

$$
\left(k-\alpha_{n}(s)\right)^{n-2} \ln \left(k-\alpha_{n}(s)\right)
$$

in the neighborhood of $\alpha_{n}(s)=k, k=0,1,2 \ldots$ Thus the amplitude has logarithmic cuts which are soft with the exception of $n=2$. (i.e., the discontinuity vanishes at the branch point.) The branch points are all at higher energies than the energy of the first resonance and can be associated with inelastic thresholds corresponding to multiresonance production.

It is perhaps worth mentioning that the structure of these cuts can be altered if one is prepared to alter the asymptotic behaviour of eqs.(2.10) and (2.11). We can insert a factor

$$
[1-\ln (x(1-x))]^{\frac{1}{2}}
$$

into $H$ and also modify $\delta$ by the insertion of $[1-\ln (x(1-x))]^{-\frac{1}{2}}$. This leads to no modification of $M_{1}(s, t)$ but does change the structure of all the other cuts. The changes are as follows.

(a) The $s$ plane cuts in $M_{2}(s, t)$ take the form of elastic-type cuts. This follows from the $x=0$ behaviour. The integral now contains an extra factor $(-\ln x)^{-\frac{1}{2}}$ which when combined with the expression (2.14) gives

$$
M_{2}(s, t) \sim\left[k-\alpha_{2}(s)\right]^{\frac{1}{2}}
$$

near $\alpha_{2}(s)=k$. Other cuts are also modified, e.g.,

$$
M_{3}(s, t) \sim\left[k-\alpha_{3}(s)\right]^{2} \ln \left(k-\alpha_{3}(s)\right) \text { etc. }
$$

(b) The Regge behaviour is also modified. Eq.(2.11) is replaced by

$$
M_{n}(s, t) \sim \frac{\Gamma\left(-\alpha_{n}(s)\right)}{\ln ^{\frac{3}{2}(n-1)}(-t)}\left(-\frac{t r}{n}\right)^{\alpha_{n}(s)} .
$$


Since it is not clear whether such factors (2.17) should be introduced we present them here as suggestions. In what follows we shall not insert them, the discussions can be easily modified if it turns out that their presence is necessary.

To discuss the analytic continuation in the presence of non-linear trajectories and to establish the boundaries of the double spectral functions generated by $M_{n}(s, t)$ it is useful to use the Bessel representation (2.5).

$M_{n}(s, t)=\frac{(2 \pi)^{2}}{n !} \int_{0}^{1} \mathrm{~d} x[x(1-x)]^{-3} \int_{0}^{\infty} b_{s} \mathrm{~d} b_{s} \int_{0}^{\infty} b_{t} \mathrm{~d} b_{t} J_{0}\left(b_{t} q_{t}\right) J_{0}\left(b_{s} q_{s}\right) \delta^{n}\left(x, b_{s}, b_{t}\right)$,

where

$$
\begin{aligned}
& \delta\left(x, b_{s}, b_{t}\right)=\frac{-\lambda}{(2 \pi)^{2}} \int_{0}^{\infty} x_{s} \mathrm{~d} x_{s} \int_{0}^{\infty} x_{t} \mathrm{~d} x_{t} J_{0}\left(b_{s} x_{s}\right) J_{0}\left(b_{t} x_{t}\right) \\
& \times x^{-\alpha_{\mathrm{R}}\left(-x_{s}^{2}\right)+2-\alpha_{\mathrm{I}}\left(-x_{s}^{2}(1-x)\right)}(1-x)^{-\alpha_{\mathrm{R}}\left(-x_{t}^{2}\right)+2-\alpha_{\mathrm{I}}\left(-x_{t}^{2} x\right)} .
\end{aligned}
$$

For simplicity we have again set $f=1$.

With the aid of the relation [17]

$$
\int_{0}^{\infty} J_{0}(b \sqrt{-t}) J_{0}\left(b \sqrt{-t_{1}}\right) J_{0}\left(b \sqrt{-t_{2}}\right) b \mathrm{~d} b=\frac{2}{\pi} \frac{\theta(-\lambda)}{\sqrt{-\lambda\left(t, t_{1}, t_{2}\right)}}
$$

where

$$
\lambda\left(t, t_{1}, t_{2}\right)=t^{2}+t_{1}^{2}+t_{2}^{2}-2 t t_{1}-2 t t_{2}-2 t_{1} t_{2},
$$

we have the following representation for $M_{2}(s, t)$ :

$$
\begin{aligned}
& M_{2}(s, t)=\frac{\lambda^{2}}{(2 \pi)^{15}} \frac{1}{\pi} \int_{-\infty}^{0} \frac{\mathrm{d} t_{1} \mathrm{~d} t_{2} \mathrm{~d} s_{1} \mathrm{~d} s_{2} \theta\left(-\lambda_{1}\right) \theta\left(-\lambda_{2}\right)}{\sqrt{-\lambda_{1}\left(t, t_{1}, t_{2}\right)} \sqrt{-\lambda_{2}\left(s, s_{1}, s_{2}\right)}} \\
& \quad \times \int_{0}^{1} \mathrm{~d} x x^{-\alpha_{\mathrm{R}}\left(s_{1}\right)-\alpha_{\mathrm{R}}\left(s_{2}\right)+1-\alpha_{\mathrm{I}}\left(s_{1}(1-x)\right)-\alpha_{\mathrm{I}}\left(s_{2}(1-x)\right)} \\
& \quad \times(1-x)^{-\alpha_{\mathrm{R}}\left(t_{1}\right)-\alpha_{\mathrm{R}}\left(t_{2}\right)+1-\alpha_{\mathrm{I}}\left(t_{1} x\right)-\alpha_{\mathrm{I}}\left(t_{2} x\right)} .
\end{aligned}
$$

The higher terms can be expressed in terms of invariants in a similar way if we use the relation

$$
J_{0}\left(r \sqrt{-t_{2}}\right) J_{0}\left(r \sqrt{-t_{3}}\right)=\frac{1}{\pi} \int_{-\infty}^{0} \mathrm{~d} t_{1} \frac{J_{0}\left(r \sqrt{-t_{1}}\right) \theta(-\lambda)}{\sqrt{-\lambda\left(t_{3}, t_{1}, t_{2}\right)}}
$$


in order to reduce the chain products of Bessel functions that occur. For example the next term yields

$$
\begin{aligned}
& M_{3}(s, t)=\frac{-\lambda^{3}}{3 !(2 \pi)^{24}} \int_{-\infty}^{0} \frac{\mathrm{d} s_{1} \mathrm{~d} s_{2} \mathrm{~d} s_{3} \mathrm{~d} s_{23}}{\sqrt{-\lambda_{1}\left(s_{2}, s_{3}, s_{23}\right)}} \frac{\theta\left(-\lambda_{1}\right) \theta\left(-\lambda_{2}\right)}{\sqrt{-\lambda_{2}\left(s, s_{1}, s_{23}\right)}} \\
& \quad \times \int_{-\infty}^{0} \frac{\mathrm{d} t_{1} \mathrm{~d} t_{2} \mathrm{~d} t_{3} \mathrm{~d} t_{23}}{\sqrt{-\lambda_{3}\left(t_{2}, t_{3}, t_{23}\right)}} \frac{\theta\left(-\lambda_{3}\right) \theta\left(-\lambda_{4}\right)}{\sqrt{-\lambda_{4}\left(t_{1}, t_{23}, t\right)}} \int_{0}^{1} \mathrm{~d} x x^{-\alpha_{\mathrm{R}}\left(s_{1}\right)-\alpha_{\mathrm{R}}\left(s_{2}\right)} \\
& \quad \times x^{-\alpha_{\mathrm{R}}\left(s_{3}\right)+3-\alpha_{\mathrm{I}}\left(s_{1}(1-x)\right)-\alpha_{\mathrm{I}}\left(s_{2}(1-x)\right)-\alpha_{\mathrm{I}}\left(s_{3}(1-x)\right)} \\
& \quad \times(1-x)^{-\alpha_{\mathrm{R}}\left(t_{1}\right)-\alpha_{\mathrm{R}}\left(t_{2}\right)-\alpha_{\mathrm{R}}\left(t_{3}\right)+3-\alpha_{\mathbf{I}}\left(t_{1} x\right)-\alpha_{\mathbf{I}}\left(t_{2} x\right)-\alpha_{\mathbf{I}}\left(t_{3} x\right) .}
\end{aligned}
$$

We can regard eq.(2.22) as a coupling scheme (with a particular choice of coupling functions) in which dual reggeons two and three are first coupled to form an effective particle which is then coupled to the third reggeon.

The basic technique for continuing eq.(2.20) has been given by Polkinghorne and Olive [18] in their analysis of Mandelstam cuts. For negative $s$ and $t$ the $\left(s_{1}, s_{2}\right)$ and $\left(t_{1}, t_{2}\right)$ integration domains are the third quadrant interiors of the parabolas $\lambda_{1}=0, \lambda_{2}=0$ in their respective planes. As $s$ and $t$ increase to positive values each domain of integration degenerates into a coincident line pair and reappears as a domain in the relevant complex plane. Using the Cauchy theorem in its generalized form we can rotate these integration domains into the real $\left(s_{1}, s_{2}\right),\left(t_{1}, t_{2}\right)$ planes again. This procedure is permissible until such values of $s$ and $t$ are encountered that yield unavoidable singularities in the integrand. (For details see ref.[18].)

Observe that there are two sources of singularities in our expression (2.20). The first is associated with the divergence of the $x$-integration leading to the previously discussed singularities. The discontinuities of the singularities are linear in the other variable and so do not contribute to the double spectral functions. The second stems from the explicit threshold singularities contained in $\alpha_{1}$. Analyzing eq. (2.20) for simplicity we see that $M_{2}$ will have a singularity in $s$ at a value $s=\left(\sqrt{ } s_{1}^{0}+\sqrt{ } s_{2}^{0}\right)^{2}$ where $s_{1}^{0}$ and $s_{2}^{0}$ are the positions of the singularities in $s_{1}$ and $s_{2}$. The singularities in $s_{1}$ and $s_{2}$ coming from $\alpha_{\mathrm{I}}$ are at

$$
s_{1}^{0}=\frac{(t h) s_{1}}{1-x}, \quad s_{2}^{0}=\frac{(t h) s_{2}}{1-x},
$$

and so taking the discontinuity over $a$ will introduce

$$
\theta\left(s-\left(\sqrt{\frac{(t h) s_{1}}{1-x}}+\sqrt{\frac{(t h) s_{2}}{1-x}}\right)^{2}\right) \rightarrow \theta\left(s(1-x)-\left(\sqrt{(t h)_{s_{1}}}+\sqrt{(t h)_{s_{2}}}\right)^{2}\right) .
$$

Taking the discontinuity over $t$ introduces 


$$
\theta\left(t x-\left(\sqrt{(t h)_{t_{1}}}+\sqrt{(t h)_{t_{2}}}\right)^{2}\right)
$$

which tells us that the support of the double spectral function* is

$$
t s-t\left(\sqrt{(t h)_{s_{1}}}+\sqrt{(t h)_{s_{2}}}\right)^{2}-s\left(\sqrt{(t h)_{t_{1}}}+\sqrt{(t h)_{t_{2}}}\right)^{2} \geqslant 0 .
$$

We end this section with some comments on the form of $H(\delta)$ which we have chosen to be expression (2.3)

$$
H(\delta)=\mathrm{e}^{\delta}-1
$$

This choice was motivated by the formal similarity of our model to the Reggeeikonal model. However, the conventional eikonalisation procedure is performed in either the $s$-channel or the $t$-channel. In our case the above choice for $H$ may not be the most appropriate. In fact in the next section we briefly discuss a model in which the freedom of choice in the direction of $\boldsymbol{q}_{s}$ and $\boldsymbol{q}_{t}$ allows us to construct an eikonalisation in both the $s$ and $t$-channel with a single two dimensional integration over the impact variable $\boldsymbol{b}$. This model is compared with eq.(2.1) and it suggests that $H(\delta)$ in eq.(2.1) should be $\delta \mathrm{e}^{\delta}$.

\section{MODEL WITH ONE IMPACT VARIABLE}

In this section we choose $\boldsymbol{q}_{S}$ and $\boldsymbol{q}_{t}$ such that

$$
\boldsymbol{q}_{s} \cdot \boldsymbol{q}_{t}=0
$$

and introduce the following integral representation

$$
M(s, t)=\int_{0}^{1} \mathrm{~d} x[x(1-x)]^{-3} \ln x \ln (1-x) \int \mathrm{d}^{2} b \mathrm{e}^{i b \cdot a} H(\delta)
$$

where

$$
\begin{aligned}
H(\delta) & =\mathrm{e}^{\delta}-1, \\
q & =q_{S}(-\ln x)^{\frac{1}{2}}+q_{t}(-\ln (1-x))^{\frac{1}{2}}, \\
\delta(x, b) & =\frac{-\lambda}{(2 \pi)} \int \mathrm{d}^{2} k \mathrm{e}^{-i b \cdot k} \frac{x^{2-a_{1}}(1-x)^{2-a_{2}} \mathrm{e}^{-r k^{2}}}{\ln x \ln (1-x)} \\
& =\frac{-\lambda}{2 r} \frac{x^{2-a_{x}}(1-x)^{2-a_{2}}}{\ln x \ln (1-x)} \mathrm{e}^{-b^{2} / 4 r} .
\end{aligned}
$$

* To obtain the correct double spectral function boundaries we can make the necessary modifications suggested in ref. [11]. 
This slightly artificial looking model reproduces the Veneziano model as the first term in the expansion of $\mathrm{e}^{\delta}-1$. The trajectories in the $s$ and $t$-channels have intercepts $a_{1}$ and $a_{2}$ and slope $r$. Since the eikonalization is performed in both $s$ and $t$-channels simultaneously we have chosen $H(\delta)=\mathrm{e}^{\delta}-1$. We observe that $M_{2}$, for example, can be written as

$$
\begin{aligned}
& M_{2}(s, t) \propto \int_{0}^{1} \mathrm{~d} x \frac{x^{2-a_{1}}(1-x)^{2-a_{2}}}{\ln x \ln (1-x)} \int_{0}^{\infty} b \mathrm{~d} b J_{0}(b \sqrt{s \ln x+t \ln (1-x))} \\
& \quad \times \int_{0}^{1} k_{1} \mathrm{~d} k_{1} J_{0}\left(b k_{1}\right) \mathrm{e}^{-r k_{1}^{2}} \int_{0}^{\infty} k_{2} \mathrm{~d} k_{2} J_{0}\left(b k_{2}\right) \mathrm{e}^{-r k_{1}^{2}} \\
& \quad \times \frac{1}{2 \pi} \int_{0}^{1} \mathrm{~d} x \frac{x^{1-2 a_{1}}(1-x)^{1-2 a_{2}}}{\ln x \ln (1-x)} \int_{0}^{\infty} \mathrm{d} k_{1}^{2} \int_{0}^{\infty} \frac{\mathrm{d} k_{2}^{2} \mathrm{e}^{-r\left(k_{1}^{2}+k_{2}^{2}\right)} \theta(-\lambda)}{\sqrt{-\lambda\left(\ln x+t \ln (1-x)_{1}-k_{1}^{2},-k_{2}^{2}\right)}} .
\end{aligned}
$$

This, perhaps surprising $\lambda$ function, reduces to the expected $\lambda$ function in the asymptotic limit. To see this take $t \rightarrow-\infty$ and introduce the variable $u=-x t$. Then

$$
M_{2}(s, t) \rightarrow \frac{(-t)^{2 a_{1}-1}}{\ln (-t)} \int_{0}^{\infty} \mathrm{d} u u^{-2 a_{1}} \int_{0}^{\infty} \mathrm{d} k_{1}^{2} \int_{0}^{\infty} \mathrm{d} k_{2}^{2} \frac{e^{-r\left(k_{1}^{2}+k_{2}^{2}\right)} \theta(-\lambda)}{\sqrt{-\lambda\left(-\sin (-t)+u,-k_{1}^{2},-k_{2}^{2}\right)}}
$$

With the further change of variables

$$
\begin{aligned}
& k_{1}^{2}=-z_{1} \ln (-t)+u, \\
& k_{2}^{2}=-z_{2} \ln (-t)+u,
\end{aligned}
$$

this becomes

$$
\begin{gathered}
M_{2}(s, t) \rightarrow(-t)^{2 a_{1}-1} \ln (-t) \int_{0}^{\infty} \mathrm{d} u u^{-2 a_{1}} \int_{-\infty}^{0} \mathrm{~d} z_{1} \int_{-\infty}^{0} \mathrm{~d} z_{2} \theta(-\lambda) \mathrm{e}^{-2 u r} \\
\times(-t)^{r z_{1}+r z_{2}}\left(-\lambda\left(-s \ln (-t)+u, z_{1} \ln (-t)-u, z_{2} \ln (-t)-u\right)\right)^{-\frac{1}{2}} \\
\rightarrow \int_{0}^{\infty} \mathrm{d} u u^{-2 a_{1}} \mathrm{e}^{-2 u r} \int_{-\infty}^{0} \mathrm{~d} z_{1} \int_{-\infty}^{0} \mathrm{~d} z_{2} \frac{\theta(-\lambda)(-t)^{2 a_{1}-1+r z_{1}+r z_{2}}}{\sqrt{-\lambda\left(s, z_{1}, z_{2}\right)}}
\end{gathered}
$$

confirming the expected two-reggeon structure.

Next we study the relation of this model to the model of sect.2. In the expression

$$
M_{n}(s, t)=\frac{1}{n !} \int_{0}^{1} \mathrm{~d} x[x(1-x)]^{-3} \ln x \ln (1-x) \int \mathrm{d}^{2} b \mathrm{e}^{i q \cdot b} \delta^{n}
$$


we shall write $\mathrm{d}^{2} \boldsymbol{b}=\mathrm{d} b_{x} \mathrm{~d} b_{y}$ and introduce new variables $B_{x}=b_{x}(-\ln x)^{\frac{1}{2}}$ and $B_{y}=$ $b_{y}(-\ln (1-x))^{\frac{1}{3}}$.

If we utilize the relation

$$
\int_{-\infty}^{\infty} \mathrm{e}^{b^{2} / \ln x} \mathrm{~d} b=\sqrt{\pi(-\ln x)}
$$

we can rewrite eq.(3.6) as

$$
\begin{aligned}
& \frac{1}{n !} \int_{0}^{1} \mathrm{~d} x(x(1-x))^{-3} \frac{n}{4 \pi r} \int \mathrm{d}^{2} b_{s} \int \mathrm{d}^{2} b_{t} \mathrm{e}^{i q_{s} \cdot b_{s}+i a_{t} \cdot b_{t}} \\
& \quad \times\left[-\frac{1}{4} \lambda \frac{x^{2-a_{1}(1-x)^{2-a_{2}}}}{\ln x \ln (1-x) r}\right]^{n} \exp \left[\frac{n}{4 r}\left(\frac{b_{s}^{2}}{\ln x}+\frac{b_{t}^{2}}{\ln (1-x)}\right)\right],
\end{aligned}
$$

where we have used the orthogonality of $\boldsymbol{q}_{s}$ and $\boldsymbol{q}_{\boldsymbol{t}}$ to introduce $\boldsymbol{b}_{s}$ and $\boldsymbol{b}_{\boldsymbol{t}}$. Thus we see that

$M(s, t)=\sum_{n=1}^{\infty} M_{n}(s, t)=\int_{0}^{1} \mathrm{~d} x[x(1-x)]^{-3} \int \mathrm{d}^{2} b_{s} \int \mathrm{d}^{2} \boldsymbol{b}_{t} \mathrm{e}^{i\left(\boldsymbol{q}_{s} \cdot \boldsymbol{b}_{s}+\boldsymbol{q}_{t} \cdot b_{t}\right)} \frac{\delta \mathrm{e}^{\delta}}{4 \pi r}$,

where

$$
\delta\left(x, b_{s}, b_{t}\right)=-\lambda \frac{x^{2-a_{1}}(1-x)^{2-a_{2}}}{4 r \ln x \ln (1-x)} \exp \left(\frac{b_{s}^{2}}{4 r \ln x}+\frac{b_{t}^{2}}{4 r \ln (1-x)}\right\} .
$$

We recognize the formulae $(2.1),(2.2)$ and $(2.3)$ with the particular choice

$$
\begin{gathered}
F=1, \\
G=1, \\
H(\delta)=\delta \mathrm{e}^{\delta},
\end{gathered}
$$

and linear trajectories.

It is interesting to investigate the $s$ behaviour at high $t$ of our model (2.1). This behaviour depends crucially on the form of $H(\delta)$ and so guided by the comparison with the model (3.2) we choose $H(\delta)$ in eq.(2.1) as $\delta \mathrm{e}^{\delta}$. Of course the details of the behaviour will also depend on the form of $F$ and $G$. We know from experiment that Regge trajectories are approximately linear so we might hope that setting $F=G=1$ will not affect our analysis too much. Since eq.(2.1) may be made equivalent to eq.(3.2) we shall choose the latter to study this behaviour. We write

$$
M(s, t)=\int_{0}^{1} \mathrm{~d} x[x(1-x)]^{-3} \ln x \ln (1-x) \int_{0}^{\infty} b \mathrm{~d} b J_{0}\left(b \sqrt{\sin x+t \ln (1-x)}\left\{\exp \left(A \mathrm{e}^{-b^{2} / c}\right)-1\right\}\right.
$$


where

$$
\begin{aligned}
& A=\frac{-\lambda x^{2-a_{1}}(1-x)^{2-a_{2}}}{4 r \ln x \ln (1-x)}, \\
& c=4 r,
\end{aligned}
$$

and notice that as a function of $b$ the expression in curly brackets above has a vanishing derivative at $b=0$ at $b=\infty$. The first derivative has an extremum at

$$
1+A \mathrm{e}^{-b^{2} / c}=\frac{c}{2 b^{2}}
$$

and since the bracket is non zero at $b=0$ the major contribution to the $b$ integral in eq.(3.10) comes from the region $b \leqslant b_{0}$ where $b_{0}$ is the solution to eq.(3.11). Since $A$ is small we have

$$
b_{0}^{2} \approx \frac{1}{2} c=2 r .
$$

Approximating the integral

$$
\int_{0}^{\infty} b \mathrm{~d} b J_{0}(b z)\left\{\exp \left(A \mathrm{e}^{-b^{2} / c}\right)-1\right\} \sim \int_{0}^{b_{0}} b \mathrm{~d} b J_{0}(b z)=\frac{b_{0}}{z} J_{1}\left(b_{0} z\right),
$$

where

$$
z=\sqrt{\sin x+t \ln (1-x)}
$$

we see that

$$
M(s, t) \approx \int_{0}^{1} \mathrm{~d} x[x(1-x)]^{-3} \ln (x) \ln (1-x) J_{1}(\sqrt{2 r} z) / z
$$

For large $t$ this gives the following $s$ behaviour for the whole expression

$$
M(s, t) \approx \beta(t) \frac{J_{1}(\sqrt{2 r} \sqrt{-s \ln (-t)}}{\sqrt{-s}} .
$$

In this limit all the $s$ dependence of the model is given in terms of the first order Bessel function dirived by $\sqrt{-s}$. The arguments above are crude and imprecise but the result is suggestive of the popular optical models of high energy $\sqrt{t}$ scattering. It is not known experimentally what $s$ behaviour the many-reggeon part of the amplitude should reproduce but in the approximation of treating the bare pomeron* as a Regge trajactory we obtain the above Bessel function as an approximation to the diffractive peaks which seem to be experimentally verified [21].

\footnotetext{
* In this language the conventional pomeron should presumably be associated with our bare pomeron plus all the multi-pomeron cuts.
} 


\section{A MODEL WITH REGGEON-MANY POMERON CUTS}

In the last two sections we studied models of amplitudes with many reggeon cuts. Phenomenologically however, reggeon-many pomeron cuts appear more important as they are expected to be stronger and always accompany Regge pole exchanges [13].

The model we propose in this case is again eq.(2.1) with an appropriate choice of $H$. We have to introduce two kinds of $\delta$ now: $\delta_{\mathrm{R}}$ corresponding to Regge poles and $\delta_{\mathrm{P}}$ to be associated with the bare pomeron. Guided by the ideas of the last section we take

$$
H\left(\delta_{\mathrm{P}}, \delta_{\mathrm{R}}\right)=\delta_{\mathrm{R}}\left(1+\delta_{\mathrm{P}} \mathrm{e}^{\delta_{\mathrm{P}}}\right)
$$

This would correspond in the one impact variable model of sect. 3 to $\delta_{\mathrm{R}} \mathrm{e}^{\delta_{\mathrm{P}}}$. For $\delta_{\mathrm{R}}$ we take expression (2.2) of sect.2. As the nature (and definition) of the pomeron singularity is not known we shall assume that it is phenomenologically reasonable to approximate the input pomeron by an effective Regge pole and so take

$$
\begin{gathered}
\delta_{\mathrm{P}}=\frac{-\lambda}{(2 \pi)^{4}} \int \mathrm{d}^{2} x_{s} \int \mathrm{d}^{2} x_{t} \mathrm{e}^{-i x_{s} \cdot b_{s}-i x_{t} \cdot b_{t}} F_{\mathrm{P}}\left(x_{s}^{2}(1-x), x_{t}^{2} x, x,(1-x)\right) \\
\times x^{-\alpha_{\mathrm{P}}\left(-x_{s}^{2}\right)+2}(1-x)^{-\alpha_{\mathrm{P}}\left(-x_{t}^{2}\right)+2} .
\end{gathered}
$$

Presumably $\alpha_{\mathrm{P}}$ should have a small slope and the approximation $F_{\mathrm{P}}=1$ is expected to be less reliable now.

The analysis proceeds as in the previous two sections. We discover

(a) Regge-pomeron cuts in the high energy behaviour.

(b) Regge-pomeron resonance-like cuts in the finite energy plane. Their interpretation is unclear although they are expected to be shielded by the threshold cuts from the Regge trajectories $\alpha_{\mathrm{R}}$.

A real difference arises when one studies the $s$ dependence of the amplitude for large $t$. Again it is convenient to go to the representation introduced in section three. With $F=1$ and linear Regge and pomeron trajectories with different slopes

$M(s, t) \propto \int_{0}^{1} \mathrm{~d} x[x(1-x)]^{-3} \ln x \ln (1-x) \int_{0}^{\infty} b \mathrm{~d} b J_{0}(b \sqrt{s \ln x+t \ln (1-x)}) \delta_{\mathrm{R}} \mathrm{e}^{\delta} \mathrm{P}$,

where

$$
\begin{aligned}
& \delta_{\mathrm{R}}(x, b)=\frac{x^{2-a_{1}}(1-x)^{2-a_{2}}}{4 r \ln x \ln (1-x)} \mathrm{e}^{-b^{2} / 2 r} \\
& \delta_{\mathrm{P}}(x, b)=\frac{x^{2-a_{\mathrm{P}}}(1-x)^{2-a_{\mathrm{P}}}}{4 r_{\mathrm{P}} \ln x \ln (1-x)} \mathrm{e}^{-b^{2} / 2 r_{\mathrm{P}}}
\end{aligned}
$$




$$
\alpha_{\mathrm{P}}(s)=a_{\mathrm{P}}+r_{\mathrm{P}} s
$$

Introducing

$$
\begin{aligned}
& \mathrm{C}=\delta_{\mathrm{P}} \mathrm{e}^{b^{2} / 2 r_{\mathrm{P}},} \\
& A=4 r, \\
& D=4 r_{\mathrm{P}},
\end{aligned}
$$

we see that the $b$ dependence of $\delta_{\mathrm{R}} \mathrm{e}^{\delta} \mathrm{P}$ is contained in the function

$$
\exp \left\{-\frac{b^{2}}{A}+C \mathrm{e}^{-b^{2} / D}\right\}
$$

The first derivative with respect to $b$ vanishes again at $b=0$ and $b=\infty$ and also at $b_{0}=[-D \ln (-D / A C)]$. Taking the major contribution to the $b$ integral in eq.(4.3) from the maximum at $b=b_{0}$

$M(s, t) \propto \int_{0}^{1} \mathrm{~d} x[x(1-x)]^{-3} b_{0} J_{0}\left(b_{0} \sqrt{\sin x+t \ln (1-x))} x^{2-a_{1}}(1-x)^{2-a_{2}}\right.$.

To obtain the behaviour for large $-t$ we change variable to $u=-x t$ and obtain

$$
\begin{aligned}
& M(s, t) \rightarrow(-t)^{a_{1}} \int_{0}^{\infty} \mathrm{d} u u^{1-a_{1}}\left\{-4 r_{1} \ln \frac{\left(-u r_{\mathrm{P}} \ln (-t)\right)}{\lambda t r}\right\}^{\frac{1}{2}} \\
& \quad \times J_{0}\left(\left\{\left[-4 r_{\mathrm{P}} \ln \frac{\left(-u \ln (-t) r_{\mathrm{P}}\right)}{\lambda t r}\right][-s \ln (-t)+u]^{\frac{1}{2}}\right\}^{\frac{1}{2}}\right) \\
& \sim(-t)^{\mathrm{a}_{1}}\{\ln (-t)\}^{\frac{1}{2}} J_{0}\left(2 \sqrt{-s r_{\mathrm{P}}} \ln (-t)\right) .
\end{aligned}
$$

This crude analysis is given only to show the kind of behaviour we expect from a more realistic analysis in which $F_{\mathrm{P}}$ is not approximated by unity.

\section{DISCUSSION}

So far we have not discussed the choice of $G$ in eq.(2.1) having set it equal to unity in previous sections. As we were discussing the case of four spinless particles. we expect to use the freedom in the choice of $G$ for the construction of more realistic models. For example the choice

$$
\begin{aligned}
& G=x(1-x)(1-\alpha(s)-\alpha(t)), \\
& F=1
\end{aligned}
$$


gives for $M_{1}$ the Lovelace formula for the scattering of pions [19].

We have not discussed the problem of introducing signature into our models. This problem is, of course, related to the problem of signature in the Veneziano model. The simple procedure of adding $M(s, u)$ and $M(t, u)$ to $M(s, t)$ does not in general lead to an acceptable signature structure for the Regge cuts [20]. This problem appears complicated and has not been solved yet.

One can also study the fixed angle behaviour of our amplitudes although this also turns out to be difficult in general and will not be attempted here.

In this paper we have discussed a class of models which generate an infinite series of Regge cuts and which, at the same time, are not too complicated to be of some phenomenological use. Although we have discussed the scattering of scalar particles coupled to unsignatured trajectories we believe that the complications inherent in physical scattering processes can be incorporated without altering the previous results in any significant way. The models have considerable freedom in terms of unspecified parameters and functions but are endowed with a number of properties that we consider to be important for an adequate description of hadron physics.

The authors would like to thank Professors Drell, Blankenbecler and Gilman for their hospitality at S.L.A.C. where most of the work reported here was carreid out. One of us (R.W.T.) would like to thank A.R. Swift and the other (W.J.Z.) H.Harari for valuable, critical discussions.

\section{REFERENCES}

[1] R. Dolen, D. Horn, C. Schmid, Phys. Rev. Lett. 19 (1967) 402 and Phys. Rev. 166 (1968) 1768 .

[2] J.D. Jackson, Rev. Mod. Phys. 42 (1970) 12;

D. Sivers, J. Yellin, Rev. Mod. Phys. 43 (1971) 125.

[3] G. Veneziano, Nuovo Cim. 57A (1968) 190.

[4] K. Kikkawa, B. Sakita, M.A. Virasoro, Phys. Rev. 184 (1969) 1701;

K. Kikkawa, S. Klein, B. Sakita, M. Virasoro, Phys. Rev. D1 (1970) 3258;

A. Neveu, J.H. Schwarz, Nucl. Phys. B31 (1971) 86.

[5] C.B. Chiu, G.C. Fox, Proc. Conf. on phenomenology of particle physics, California Institute of Technology, Pasadena (1971).

[6] F. Henyey, G.L. Kane, J. Pumplin, M.H. Ross, Phys. Rev. 182 (1969) 1579;

G.L. Kane, F. Henyey, D.R. Richards, M. Ross, G. Williamson, Phys. Rev. Lett. 25 (1970) 1519.

[7] R.C. Arnold, M.L. Blackman, Phys. Rev. 176 (1968) 2082;

C. Lovelace, Nucl. Phys. B12 (1969) 253.

[8] D. Amati, S. Fubini, A. Stanghellini, Nuovo Cim. 26 (1962) 896.

[9] R.C. Arnold, Phys. Rev. 153 (1967) 1523;

R. Glauber, Lectures in theoretical physics, Boulder, vol.I. (1958).

[10] K. Kikkawa, Phys. Rev. 187 (1969) 2249.

[11] G. Cohen-Tannoudji, F. Henyey, G.L. Kane, W.J. Zakrzewski, Phys. Rev. Lett. 26 (1971) 112. 
[12] A.R. Swift, R.W. Tucker, Phys. Rev. D1 (1970) 2914;

D.D. Coon, Phys, Rev. 186 (1969) 1422;

R. Ramachandran, M.O. Taha, preprint 1C-71-30, Trieste.

[13] H. Harari, Phys. Rev. Lett. 26 (1971) 1079.

[14] P. Olesen, preprint CERN Th-1322;

M.G. Schmidt, preprint Heidelberg;

A.I. Bugrij, L.L. Jenkowsky, N.A. Kobylinsky, preprint ITP 71-28E.

[15] M. Suzuki, Phys. Rev. Lett. 23 (1969) 203.

[16] Handbook of mathematical functions, ed. M. Abramowitz, I. Stegun (National Bureau of Standards)p. 262.

[17] Tables of integrals, series and products, I.S. Gradshteyn, I.M. Ryzhik, Academic Press N.Y., p. 696.

[18] D.I. Olive, J.C. Polkinghorne, Phys. Rev. 171 (1968) 1475.

[19] C. Lovelace, Phys. Lett. 28B (1968) 265.

[20] D. Branson, Phys. Rev. 179 (1969) 1608.

[21] F. Zachariasen, Preprint CERN Th. 1284. 